ORIGINAL ARTICLE

\title{
Proposed risk stratification in upper gastrointestinal haemorrhage: Is hospitalisation essential?
}

\author{
A E Courtney, R M S Mitchell, L Rocke, B T Johnston
}

Emerg Med J 2004;21:39-40. doi: 10.1136/emj.2003.012328

See end of article for authors' affiliations

....................

Correspondence to:

Dr B T Johnston,

Department of

Gastroenterology, Royal

Victoria Hospital,

Grosvenor Road, Belfast

BT12 6BA, UK; brian.

johnston@royalhospitals.

n-i.nhs.uk

Accepted for publication 30 October 2003

\begin{abstract}
Aims: Patients with upper gastrointestinal haemorrhage (UGIH) are usually admitted to hospital regardless of the severity of the bleed. The aim of this study was to identify patients who could be safely managed without hospitalisation and immediate inpatient endoscopy.

Methods: Based on a literature review, a protocol was devised using clinical and laboratory data regarded as being of prognostic value. A retrospective observational study of consecutive patients who attended the emergency department (ED) with UGIH was conducted during one calendar month.

Results: Fifty four patients were identified of whom 44 (81\%) were admitted. Twelve suffered an adverse event. One of the 10 patients (10\%) initially discharged from the ED was later admitted. Strict implementation of the protocol would have resulted in safe discharge of a further 15 patients, (34\% of those admitted), and a saving of an estimated 37 bed days per month.

Conclusions: Patients at low risk from UGIH may be identified in the ED. If validated, this protocol may improve patient management and resource utilisation.
\end{abstract}

U pper gastrointestinal haemorrhage (UGIH) is a common medical emergency with an incidence of about 100 per 100000 adults per annum in the UK. ${ }^{1}$ The clinical severity ranges from insignificant bleeds to catastrophic exsanguination. Hospital admission is generally regarded as obligatory, although a substantial proportion of patients are at very low risk of re-bleeding or death. ${ }^{12}$ Early identification of "low risk" patients may permit safe outpatient management and lead to major resource savings. Scoring systems that are usually based on clinical and endoscopic data have been successful in predicting patients at high risk of re-bleeding and death. The system proposed by Rockall identifies independent predictors of mortality and has been prospectively validated. ${ }^{2-5}$ However, if urgent endoscopy is not widely available these systems are of little practical value. Accurate risk stratification based on clinical and laboratory data alone is not well established.

Our aim was to design a protocol based on clinical and laboratory findings at presentation that safely and accurately identifies patients at minimal risk from UGIH, thereby facilitating discharge from the emergency department (ED) without the need for upper GI endoscopy. We performed a retrospective, observational study of patients with UGIH to assess the potential quality of care and resource implications of implementing this protocol in the ED.

\section{METHODS}

A protocol was designed after a detailed literature review of validated prognostic factors of severity of UGIH (boxes 1 and 2 ). The medical records of consecutive patients presenting to the ED with UGIH during one month were reviewed. Patient characteristics, symptoms, medications, past history, examination findings, laboratory results, placement (admission or discharge), and subsequent clinical course were recorded. Adverse events were defined as transfusion requirement, need for surgical intervention, need for intensive care support, and inhospital death. All admissions and laboratory tests ordered from ED were monitored for the following month to ascertain re-attendance of discharged patients. This was performed for both Belfast hospitals responsible for alternate day take-in (Royal Victoria Hospital and Belfast City
Hospital).The potential total bed day savings were calculated assuming the protocol had been strictly applied to every patient.

\section{RESULTS}

Fifty four patients presenting to the ED with UGIH were identified. Thirty three $(61 \%)$ were male. The mean age was 49 years (range 16-91). Forty four patients (81\%) were hospitalised. Eleven patients $(20 \%)$ required blood transfusion, one patient required emergency surgical intervention, one patient was admitted to the intensive care unit because of concurrent trauma, and one patient died because of acute left ventricular failure. One patient, initially discharged from the ED, was subsequently admitted after re-presenting to the ED.

Adverse events occurred in 7 of 12 (58\%) patients presenting with melaena, in four of four patients (100\%) with a history of oesophageal varices, in one of three patients (33\%) with a systolic blood pressure of less than $100 \mathrm{~mm} \mathrm{Hg}$ at presentation, and in 10 of 15 patients $(67 \%)$ with initial haemoglobin of less than $120 \mathrm{~g} / \mathrm{l}$.

If our protocol had been strictly applied to every patient, all of the patients who suffered an adverse event would have been admitted on their initial presentation, 15 patients who were admitted would have been safely discharged from the ED, and two patients who were initially discharged would have been admitted (one of the latter patients was the patient who re-presented after being discharged from the ED). We estimate that up to 37 bed days could have been saved in the study month if the protocol had been strictly applied.

\section{DISCUSSION}

Although reported mortality rates attributable to UGIH have remained unchanged $(5 \%-12 \%)$ over many decades, ${ }^{1}$ bleeding stops spontaneously in most cases and requires no further intervention. However, it is difficult to reliably identify

Abbreviations: $E D$, emergency department; $U G H$, upper gastrointestinal haemorrhage 
Box 1 Conditions to be fulfilled for safe discharge of UGIH patients (based on reviews of references $1-7$ )

- Age $<60$ years

- No melaena

- No large volume red haematemesis (that is, not greater than a mouthful of blood)

- No history of varices or liver damage

- Systolic blood pressure $>100 \mathrm{~mm} \mathrm{Hg}$

- No orthostatic change in systolic blood pressure

- Haemoglobulin $>120 \mathrm{~g} / \mathrm{l}$

- Absence of significant co-morbidity (see box 2)

patients at low risk of re-bleeding or death on initial presentation; therefore, hospital admission is widely regarded as mandatory for most patients with UGIH. As a consequence, substantial resources are currently used on the inpatient management of very "low risk" patients. Risk stratification using a combination of clinical and endoscopic findings has been well validated as a means of predicting the risk of re-bleeding and inhospital mortality. ${ }^{45}$ This has been useful in identifying patients who require urgent intervention and intensive care support. There is also a need to safely identify patients at very low risk of re-bleeding or death, ideally without endoscopy, an expensive and limited resource within the United Kingdom and elsewhere.

Blatchford et al devised and prospectively evaluated a risk stratification score for UGIH without using endoscopic data. ${ }^{67}$ Regression analysis was used to retrospectively identify clinical and laboratory factors predictive of the need for endoscopic therapy in a large cohort of patients presenting with UGIH to several units in Scotland. A fast track system was then prospectively evaluated to determine if patients could be safely managed without endoscopy. They reported a sensitivity of $99 \%$ and specificity of $32 \%$ for serious bleeding. ${ }^{7}$ Age did not form part of this protocol, which is somewhat surprising given the importance of advancing age in other risk stratification studies.

The attractiveness of the protocols devised by Blatchford and ourselves is that diagnostic endoscopy is not required to make a risk assessment, an important issue in many centres in the United Kingdom that may have limited access to urgent endoscopy facilities. Patients classified as low risk could safely be discharged from the ED and susbsequently undergo diagnostic endoscopy as an outpatient. Nevertheless, several issues merit further comment. Unlike Blatchford et $a l^{67}$ we have included age under 60 years as part of our protocol in line with Rockall et $a l^{3}$ who found advancing age to be a robust independent risk factor for adverse events after UGIH. We have omitted other factors that are often regarded as clinically important, such as isolated vomiting of fresh blood. We somewhat arbitrarily considered more than a mouthful of fresh blood to be significant, although the volume can be difficult to quantify as patients will often overestimate the amount of blood vomited. However, in our study, no patients who only vomited fresh blood had an
Box 2 Significant co-morbidity (based on review of references 1-7)

- Cardiovascular: ischaemic pain, congestive cardiac failure; arrhythmias*

- Respiratory; acute failure; pneumonia, chronic obstructive pulmonary disease*

- Hepatic: cirrhosis, acute hepatitis

- Renal: dialysis dependence

- Neurological: dementia, delirium, cerebrovascular event $<6$ months

- Other: malignancy, sepsis

*Symptomatic and requiring additional treatment

adverse event. The remaining criteria in our protocol have been well validated in the studies cited.

Routine admission of all patients who present with UGIH represents a substantial consumption of resources. Strategies for achieving safe early discharge of patients are welcome, and if very low risk patients can be identified in the ED and outpatient management initiated it may be possible to avoid many costly and prolonged hospitalisations altogether. This is of advantage both to patients who are discharged from ED and those who are admitted. Those safely discharged can feel reassured and can avoid unnecessary hospitalisation. Those admitted will not run the risk of being lumped as a group with minor haematemesis. In consequence, they should receive more careful attention and monitoring. We have designed a straightforward, operator friendly protocol based on a comprehensive review of the current literature that may allow physicians to safely discharge patients with low risk UGIH from the ED and avoid hospitalisation. However, prospective evaluation including a large number of patients will be undertaken before implementation of this policy in our hospital.

\section{Authors' affiliations}

A E Courtney, R M S Mitchell, B T Johnston, Department of

Gastroenterology, Royal Victoria Hospital, Belfast, UK

L Rocke, Department of Emergency Medicine, Royal Victoria Hospital

\section{REFERENCES}

1 Rockall TA, Logan RF, Devlin HB, et al. Incidence of and mortality from acute upper gastrointestinal haemorrhage in the United Kingdom. BMJ 1995:311:222-6.

2 Longstreth GF, Feitelberg SP. Outpatient care of selected patients with acute non-variceal upper gastrointestinal haemorrhage. Lancet 1995;345:108-11.

3 Rockall TA, Logan RF, Devlin HB, et al. Selection of patients for early discharge or outpatient care after acute upper gastrointestinal haemorrhage. Lancet 1996;347: 1138-40.

4 Tham KY, Kimura H, Nagurney T, et al. Retrospective review of emergency department patients with non-variceal upper gastrointestinal haemorrhage for potential outpatient management. Acad Emerg Med 1999;6:196-201.

5 Vreeburg EM, Terwee CB, Snel P, et al. Validation of the Rockall risk scoring system in upper gastrointestinal bleeding. Gut 1999;44:331-5.

6 Blatchford O, Davidson LA, Murray WR, et al. Acute upper gastrointestinal haemorrhage in west of Scotland: case ascertainment study. BMJ 1997;315:510-14.

7 Blatchford O, Murray WR, Blatchford M. A risk score to predict the need for treatment for upper gastrointestinal haemorrhage. Lancet 2000;356:1318-21. 